\title{
Delayed auditory brainstem responses in diabetes mellitus
}

\author{
M W D O ALD, C E BIRD, J S LAWSON, F J J L TEMENDIA, \\ T N MONGA, D H C SURRIDGE, P VARETTE-CERRE, \\ D L W I L I A M S, D M L W I LLIAMS, A N D D L WILSON
}

From the Department of Psychology and Faculty of Medicine, Queen's University, Kingston, Ontario, Canada

S UMMARY Diabetic patients have longer interpeak latencies in the brainstem auditory evoked responses than age-matched controls. The delay is not related to clinical hearing loss or blood glucose level at time of testing. Since waves I and II are normal in latency, the conduction velocity of the eighth nerve is not involved. The delay occurs between waves II and V, which would reflect altered transmission times in auditory brainstem and midbrain structures, and suggests the presence of a central neuropathy in patients with diabetes.

It is well known that diabetic patients develop peripheral and autonomic neuropathy. Recent reviews ${ }^{1-3}$ have suggested that they may also suffer from central neuropathy, or degeneration of the higher nervous system; De Jong ${ }^{4}$ has pointed to clinical and pathological evidence that the brain parenchyma might be affected. Kent ${ }^{5}$ has argued that diabetic patients show some neurological and psychological symptoms that might signify premature aging. However, physiological evidence of central nervous system dysfunction has not been forthcoming, and relatively little attention has been paid to the possibility of central neuropathy in such patients.

In a pilot study $^{6} 7$ we compared the auditory cortical evoked responses of a group of diabetics with those of an age-matched control group, and found the diabetics to have greater suppression of the cortical auditory evoked response at high rates of stimulation, implying a slowing of the recovery process in the central nervous system. The interpretation of these changes was not clear; similar modifications of the auditory evoked response could occur in clinical hearing loss, which has been reported in diabetics. ${ }^{8-12}$ Hypoglycaemia,

Address for reprint requests: Dr MW Donald, Department of Psychology, Queen's University, Kingston, Canada K7L 3N6.

Accepted 18 May 1981 which produces a slowing of the EEG and concomitant changes in the evoked response, ${ }^{13} 14$ constitutes a further uncontrolled factor which might have produced these evoked response alterations.

We present the results of a controlled study in which audiometry was carried out in the diabetic patients to determine any peripheral hearing loss and blood glucose was estimated at the time of recording. The brainstem auditory evoked response (BAER) was sampled, rather than the longerlatency cortical responses, since the former is less vulnerable to momentary fluctuations in arousal, and afforded us an opportunity of evaluating conduction speed in the peripheral as well as central auditory system.

\section{Methods and sample}

The diabetic sample consisted of 20 insulintreated patients selected from the Diabetic Clinic of Kingston General Hospital. Their ages ranged from 20 to 60 years, with a mean of 38 years. Eleven patients were male, and nine were female. Length of illness ranged from 6 months to 45 years, with a mean duration of 16 years. Patients were excluded if they suffered from any concurrent disease which might affect the brain or the nervous system, such as uraemia due to nephropathy. No patient in the sample 
was being treated with methyldopa, nitrofurantoin, reserpine, or any medication which might be expected to interfere with the functioning of the central nervous system. Age-matched controls were obtained from the Kingston area. Six of the controls were male, and 14 were female. Their ages ranged from 21 to 61 years, with a mean of 36 years. None had a history of diabetes, all reported normal hearing, and none were taking regular medication which could be expected to affect cortical functioning. The hearing of the diabetic patients was assessed in the Department of Audiology of Kingston General Hospital. Patients were examined otoscopically, followed by audiometry to rule out peripheral hearing loss. Audiometric tests included air and bone conduction thresholds for pure tones in both ears, followed by tests of speech reception and speech discrimination in both ears. Impedance audiometry, including tympanometry and contralateral and ipsilateral reflex measurement, was also performed.

The BAER is produced by the electrical events occurring along the auditory pathway in the brain stem evoked by repeated presentation of a stimulus. Following amplification and the removal of background electroencephalogram activity these responses are averaged. Recordings were obtained in a shielded, sound-attenuated room, using an optically-isolated Nicolet CA-1000 clinical averaging system. The stimulus was a $100 \mu \mathrm{s}$ rarefaction click, delivered $65 \mathrm{db}$ above the subject's threshold, as determined by the method of limits using the same stimulus used in recording the BAER. The rate of stimulation was set at $11 \cdot 1$ per second. Percutaneous $\mathrm{Ag}-\mathrm{Ag} \mathrm{Cl}$ electrodes were fastened to the vertex $\left(C_{z}\right)$ and both mastoids, with the common electrode always being placed on the mastoid contralateral to the ear stimulated. Amplifier bandwidth was $150-300 \mathrm{~Hz}$. Averaging epochs were set at $10 \mathrm{~ms}$, with a digitising rate of 25 samples per ms. The audiometric assessment was carried out just before the patient had lunch on the same day the BAER was measured. The latter was done half an hour after lunch and, immediately following its completion, a blood sample was drawn from a vein in the patient's arm for 90 minute post cibum blood glucose estimation.

\section{Results}

The clinical audiometric data revealed no significant hearing loss in the diabetic sample. The average click thresholds for the diabetic and normal groups were not significantly different, being 15.9 and $13.7 \mathrm{db}$ and 11.9 and $11.5 \mathrm{db}$ for the right and left ears respectively. A sample of the raw data for one subject is given in the figure. The BAER was quantified in the conventional manner ${ }^{15}$ with base-to-peak amplitude and latency assessed for waves I to V. In addition the interpeak latencies I-III and I-V were determined. The morphology of the BAER was normal in both groups.

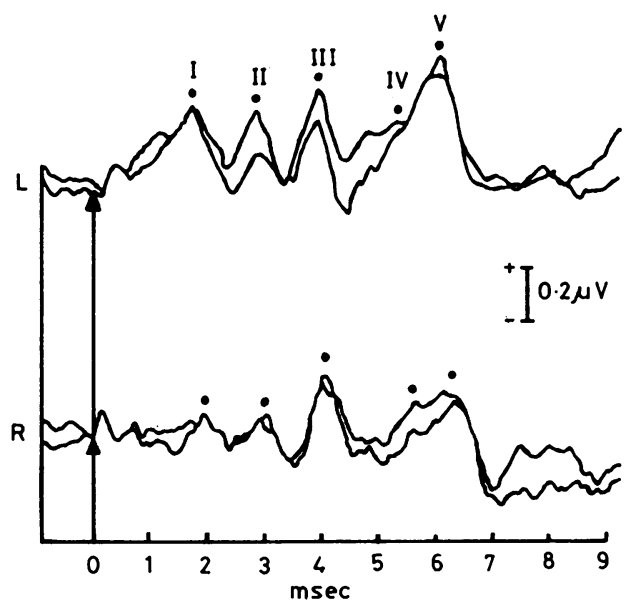

Figure Raw data for one diabetic female subject. Dots indicate latencies of waves $I-V . L=t w o$ superimposed runs for the left ear; $R=t w o$ superimposed runs for the right ear. The greatest delays in this subject were in the latency of wave $V$, and in the interpeak latency $1-V$. Stimulation rate was $11 \cdot 1 \mathrm{~Hz}$, with an EEG bandpass of $150-3000 \mathrm{~Hz}$, and 1000 repetitions per average. Vertex-to-mastoid recordings.

Table Mean latencies of all BAER variables, and mean amplitudes of waves $I V$ and $V$. Standard deviations follow mean values

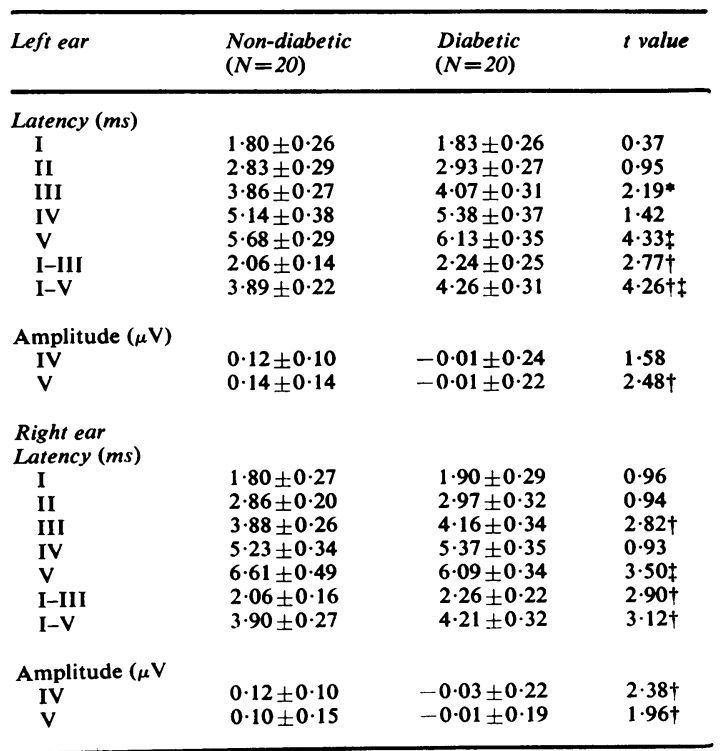

${ }^{*} p=-0.05 ; \dagger=p-0.01 ; \ddagger=p-0.001$. 
The BAER values obtained from our normal control group were compared with available published data and found to be in close agreement. ${ }^{16} 17$

The table illustrates the independent $t$ test outcomes for the group comparisons of all BAER variables. The same five measures were significantly altered in the diabetics with both right and left ear stimulation: the latencies of waves III and $\mathrm{V}$, the interpeak latencies I-III and I-V, and the amplitude of wave $\mathrm{V}$. With right ear stimulation, wave IV was also altered in amplitude. The latencies of waves I and II and the amplitudes of waves I, II, and III were not significantly different from those of the normal group, for the stimulation of either ear.

The table illustrates that all affected components were delayed, that is, longer in latency, in the diabetic patients. Since the latencies of waves III and $\mathrm{V}$ are the sum of the latencies of wave $\mathrm{I}$ and interpeak latencies I-III and I-V, it is clear that the primary effect is the delay in the interpeak latencies, which are approximately $10 \%$ longer than normal. The amplitudes of waves IV and V are reduced in the diabetic group. The average reduction is $0.12 \mu \mathrm{V}$.

The possible effect of sex differences in these results was determined with a two-way analysis of variance of the four critical BAER variables, the latencies of waves III and V and interpeak latencies I-III and I-V. The analysis strongly confirmed the effect of diabetes, and failed to find sex differences on these variables that were consistent for both ears. The only significant sex difference was in the latency of I-III in the right ear, which was shorter for females $(F(1)=4 \cdot 49$, $\mathrm{p}<0.05)$. There was no significant interaction between sex and diabetes; examination of group averages revealed that the magnitude of the latency delay in the diabetics was roughly similar in males and females.

Product-moment correlations were calculated between each BAER variable and age, duration of diabetes, and the blood sugar measurements. In the normal controls, the interpeak latencies I-III and I-V were positively correlated with age in the case of both right and left ear stimulation. In the diabetics the same variables were also correlated with age, but only for left ear stimulation; the corresponding right ear variables approached a significant correlation with age. No BAER variable was significantly correlated with duration of diabetes or with the blood glucose measurements. None of the patients in the sample was hypoglycaemic at the time of evoked potential recording.

\section{Discussion}

The delay in interpeak latencies I-III and I-V, and the reduction in amplitude of wave $V$, are evidence of a central conduction delay at the brainstem-to-midbrain level. The fact that the latencies of waves I and II were the same in both groups suggests that eighth nerve transmission time was normal in the diabetics; the delay in waves III and V, therefore, must implicate higher structures, probably at the collicular level. ${ }^{16}$

The absence of a correlation between BAER variables and blood glucose in the diabetics would appear to rule out subclinical hypoglycaemia as a source of the delay in transmission time. The duration of diabetes showed no significant relation to the BAER but this study included only one case of less than 3 years duration.

The absence of significant sex differences on the latency of wave $\mathrm{V}$ contradicts the findings of Beagley and Sheldrake ${ }^{19}$ who reported shorter latencies for wave $\mathrm{V}$ in females. Although female mean latencies in this study were shorter for waves III and V, the $F$ values did not reach significance. The differences in outcome may be due to the more conservative statistical procedure used in this study. The two-way analysis of variance procedure used in this experiment partialled out the effect of diabetes from the possible contribution of sex differences, and demonstrated clearly that sex differences did not contribute to the differences between the diabetics and nondiabetics; if anything, they might have reduced the group differences, since there were more females in the diabetic group.

The observed delay in central transmision time in diabetics may be related to the pathological observations of Reske-Nielsen and Lundbaek. ${ }^{18}$ They examined three long-term diabetics and found diffuse degeneration of the ganglion cells and nerve fibres of the cerebrum, brainstem, and cerebellum-severe enough histologically to justify the use of the term "encephalopathy". De Jong ${ }^{4}$ in reviewing their evidence, predicted that "detailed psychologic and neurologic testing of persons with longstanding diabetes mellitus might show evidence of hitherto unrecognised cerebral involvement". The results of this study confirm that prediction, at least with regard to the involvement of brainstem and midbrain regions.

The cognitive and emotional consequences of diabetic encephalopathy, or central neuropathy, are unknown. It is also not known whether the existence of such a disorder would be related to the sccurrence of peripheral neuropathy in the 
same patient. The reversibility or permanence of the disorder, and its possible relation to blood sugar regulation in diabetes, remain as yet unexplored.

\section{References}

1 Tavormina JG, Kastner LS, Slater PM, Watts SL. J Abnorm Child Psychol 1976; 4(2):99-110.

2 Shagan BP. Med Clin North Am 1976; 60(6): 1209-11.

3 Jadzinsky M, Faerman I, Fox D. Acta Diabetol Lat 1973; X:208-60.

4 De Jong RN. Postgrad Med 1977; 61(1):101-7.

5 Kent S. Geriatrics 1976; 31:140-51.

6 Lestmendia FJJ, Surridge DHC, Donald MW, et al. Ann $R$ Coll Phys Surg Canada 1979; 12(1): 81. (Abstract)

7 Donald MW, Bird CE, El-Sawy $R$ et al. In: Kornhuber $\mathrm{H}$ and Deecke $\mathrm{L}$, eds. Progress in Brain Research, Vol 54. Amsterdam: Elsevier 1980, 516-21.
8 Kofanov RL. Vestn otorinolaringol 1972; 34(3): 29-33.

9 Friedman SA, Schulman RJ, Weiss S. Arch Intern Med 1976; 135:573-6.

10 Rasic M, Rasic J, Hibsen M, Sudar A. Lyscnick Vjesnik 1977; 99(2):114-7.

11 Minami Y, Minami Y, Hori A. J Otol Japan 1977; 80(4):355-65.

12 Snashall SE. Arch Otolaryngol 1977; 103:342-3.

13 Baumann H, Baumann R, Lauter J. Acta Biol Med Ger 1969; 23:145-71.

14 Feise G, Kogure K, Busto R, Scheinburg P, Reinsmith OM. Brain Res 1976; 126:263-80.

15 Starr A, Achor LJ. Arch Neurol 1975; 32:761-8.

16 Stockard JJ, Rossiter VS. Neurology 1977; 27: 316-25.

17 Chiappa KH, Gladstone KJ, Young RR. Arch Neurol 1979; 36:81-7.

18 Reske-Nielsen E, Lundbaek R. Diabetologia 1968; 4:34-43.

19 Beagley HA, Sheldrake JB. Br J Audiol 1978; 12: 69-77. 